\title{
El jaguar Panthera onca (Carnivora: Felidae) en la Reserva de la Biosfera "El Cielo", Tamaulipas, México
}

\author{
Rogelio Carrera-Treviño ${ }^{1}$, Iván Lira-Torres ${ }^{2 *}$, Luis Martínez-García ${ }^{1} \&$ \\ Martha López-Hernández ${ }^{3}$
}

1. Laboratorio de Fauna Silvestre, Facultad de Medicina Veterinaria y Zootecnia, Universidad Autónoma de Nuevo León, Campus Ciencias Agropecuarias, Francisco Villa s/n, Col. Ex-Hacienda "El Canadá”, Escobedo, Nuevo León, México, C.P. 66050; rogeliocarrera@hotmail.com, martinez.garcialuisfer@gmail.com

2. Instituto de Ecología Aplicada, Universidad Autónoma de Tamaulipas, Av. División del Golfo No. 356. Col. Libertad, Cd. Victoria, Tamaulipas, México, C.P.87019; ilira_12@hotmail.com*

3. Reserva de la Biosfera "El Cielo", Comisión Nacional de Áreas Naturales Protegidas (CONANP), Región Noroeste y Sierra Madre Oriental. Calle Hidalgo S/N, Gómez Farías, Tamaulipas, C.P. 89780; martha.lopez@conanp.gob.mx * Correspondencia

Recibido 18-XI-2015. Corregido 23-V-2016. Aceptado 24-VI-2016.

\begin{abstract}
The jaguar Panthera onca (Carnivora: Felidae) in "El Cielo" Biosphere Reserve, Tamaulipas, Mexico. Information on the ecology of jaguars (Panthera onca) in "El Cielo" Biosphere Reserve in Tamaulipas, Mexico is scant and limited to anecdotic records in a handful of publications. The objectives of our study were to: a) determine population density and structure of jaguars, b) compare their activity patterns with that of pumas (Puma concolor), c) ascertain potential prey relative abundance, and d) evaluate local resident's perception on loss of domestic animals due to jaguar predation. Between April 2013 and April 2014 we conducted camera trapping in Gomez Farias Township with a total sampling effort of 8580 camera trap days. Besides, we completed 136 semi-structured interviews among local residents of Gomez Farias and Llera Townships to gather information on domestic animal losses attributed to jaguars and other carnivores. We identified eight different jaguar individuals during a complete year of camera-trapping, composed of four adult females, one juvenile female, two adult males and one juvenile male. We estimated a jaguar density of $5.9 \pm 1.3$ jaguars $/ 100 \mathrm{~km}^{2}$. Activity patterns for jaguars and pumas were similar as both were nocturnal and crepuscular in nature. The most abundant potential prey species for jaguars in the study site were Crax rubra, Cuniculus paca, Mazama temama, Odocoileus virginianus and Didelphis virginiana; while the rarest were Mephitis macroura and Procyon lotor. Interview results suggested that chickens, dogs, and house cats were the most consumed domestic animals from all reported losses by local residents $(n=107)$. This study represents the first attempt to describe jaguar ecology in "El Cielo" Biosphere Reserve; however, there is a need of additional monitoring efforts to determine the current status of jaguars in a larger area in order to establish conservation strategies. Finally, this jaguar population may have an important role in maintaining the species in the Sierra Madre Oriental biological corridor connecting populations in Nuevo Leon and San Luis states in Northeastern Mexico. Rev. Biol. Trop. 64 (4): 1451-1468. Epub 2016 December 01.
\end{abstract}

Key words: camera-trapping, density, activity patterns, "El Cielo" Biosphere Reserve, Panthera onca.

El jaguar Panthera onca (Carnivora: Felidae) es el carnívoro que más impacta a los animales domésticos por depredación en zonas tropicales (Zimmermann, Walpole, \& Leaderwilliams, 2005; Zeller, 2007; RosasRosas, 2006; Peña-Mondragón, 2011), es muy probable que por esta razón ha desaparecido en más del $60 \%$ de su distribución original en México (Hoogesteijn \& Hoogesteijn, 2001; Ceballos et al., 2006). Actualmente, se registra en la lista roja de especies amenazadas de la Unión Internacional para la 
Conservación de la Naturaleza (IUCN) (Caso et al., 2008), y en México se considera en peligro de extinción según la NOM-059-ECOL-2010 (SEMARNAT, 2010).

De hábitos nocturnos, principalmente terrestre y solitario, es una especie muy sigilosa y escurridiza, por lo que es uno de los animales más difíciles de estudiar (Galindo-Leal, 2009; Moreira, García, McNab, Ponce-Santizo, Mérida, \& Ruano, 2009; Chávez \& Ceballos, 2014). Históricamente, se distribuía desde Arizona y Nuevo México, en el sur de Estados Unidos hasta Argentina (Seymour, 1989). En la actualidad, su distribución en México abarca algunas de las regiones tropicales, subtropicales y templadas de Sonora y el centro de Nuevo León, hasta Chiapas y la península de Yucatán, en ambos casos siguiendo la vertiente del Golfo de México a través de la Sierra Madre Oriental y por la planicie costera del Pacífico (Carrera, Cavazos, Briones-Salas, \& Lira-Torres, 2015; Chávez-Tovar, Aranda, \& Ceballos, 2006). Las poblaciones mejor estudiadas se encuentran en el sureste del país: Campeche, Yucatán y Quintana Roo (Chávez, Ceballos, Medellín, \& Zarza, 2007; Rodríguez-Soto et al., 2011).

En el Programa de Acción para la Conservación de la Especie (PACE): Jaguar (Panthera onca), documento rector de la Dirección de Especies Prioritarias de la Comisión Nacional de Áreas Naturales Protegidas (CONANP), se describe las metas, estrategias y acciones a implementar para la conservación del jaguar en México, el Estado de Tamaulipas es considerado Nivel II entre los sitios prioritarios, debido a que es un Estado que presenta extensiones considerables de hábitat adecuado para el jaguar, pero no se ha evaluado en forma sistemática la presencia de la especie, ni se han realizado estudios ecológicos de estas poblaciones (CONANP, 2009).

Desafortunadamente, los estudios con jaguares en vida silvestre son complicados y oneroso, incluso a niveles tan elementales como conocer su presencia en un sitio determinado (Briones-Salas, Lavariega, \& Lira-Torres, 2012). Está documentado que en aquellos sitios en los que continúa presente, la densidad de sus poblacionales tiende a disminuir debido a la persecución que deriva de la hostilidad de la gente (Rabinowitz \& Nottingham, 1986; Weber \& Rabinowitz, 1996; Sanderson et al., 2002). Por lo que, para obtener registros confiables de su presencia, es necesario el uso de diferentes dispositivos de captura o registro (Gittleman, Funk, Macdonald, \& Wayne, 2001; BareaAzcón, Virgós, Ballesteros-Duperón, Moleón, \& Chirosa, 2007).

Un sitio reconocido a nivel mundial por ser una área de transición entre dos grandes zonas biogeográficas; Neártica y Neotropical, y ser refugio de importantes especies de flora y fauna silvestre, entre ellas el jaguar, es la Reserva de la Biosfera "El Cielo" (SánchezRamos, Reyes-Castillo, \& Dirzo, 2005). Está área natural se encuentra localizada en la Sierra Madre Oriental al suroeste de Tamaulipas, la cual presenta una superficie de 144530 ha pertenecientes a cuatro municipios, Gómez Farías, Ocampo, Llera y Jaumave, en los ramales conocidos como Sierra de Cucharas y Sierra Chiquita (Sánchez-Ramos et al., 2005). El Bosque Mesófilo de Montaña, bosque de niebla o nubliselva (cloud forest), fue el detonante para la creación de esta Reserva de la Biosfera, ya que este tipo de ecosistema es considerado en estado crítico de conservación de acuerdo al World Conservation Monitoring Center (Centro de Monitoreo de la Conservación) (Ramamoorthy, Bye, Lot, \& Fa, 1993). Solo en México se estima que el $5 \%$ de estos fragmentos presenta algún tipo de protección, siendo este tipo de vegetación el más raro del país $(<1 \%$ del territorio nacional) (Toledo, 1988; Vales, Rodríguez, De la Maza, Gonzáles, \& Bretón, 2000; Vidal, 1999).

La Reserva de la Biosfera "El Cielo" resulta de vital importancia en la conservación de las poblaciones de jaguares de la vertiente del Golfo de México, ya que funciona como un corredor biológico que conecta las poblaciones de San Luis Potosí con las poblaciones del centro de Nuevo León (López-Soto, Rosas-Rosas, \& Niño-Ramírez, 1997; Grigione et al., 2009; Dueñas-López, 2013). No obstante, ante la creciente problemática de pérdida y fragmentación 
del hábitat de esta región por actividades agropecuarias, esta especie se encuentra en peligro de extinción (Caso, 2007). Aunado a lo anterior, la eliminación de ejemplares en represalia por ataques a animales domésticos representa otra gran amenaza (Swank \& Teer, 1989; Chávez \& Ceballos, 2014). Al respecto, Caso (2007) menciona que la presencia de jaguares en la cabecera municipal de Gómez Farías es esporádica, y los lugareños se quejan más por los daños en su ganadería por oso negro (Ursus americanus) que por los daños de los felinos mayores (puma y jaguar). Esto es contrastante, ya que en los últimos años se han incrementado los reportes de depredación de animales domésticos por jaguares (CONANP, 2013), lo que ha llamado la atención de residentes locales y alertado a las autoridades municipales y estatales.

El conflicto generado por la depredación de animales domésticos por carnívoros y la consecuente eliminación de estos no es algo nuevo, por lo que es considerado como una de las amenazas que genera más presión a las poblaciones de estas especies alrededor del mundo, al mismo tiempo de que es un problema que causa pérdidas económicas importantes a diversas comunidades y dueños de tierra (Woodroffe \& Ginsberg, 1998). La gran mayoría de los casos por depredación de animales domésticos refleja algún tipo de desequilibrio ecológico en el área: pérdida y fragmentación del hábitat, disminución de presas potenciales, aumento de las poblaciones humanas, asentamiento de las mismas en los bordes de las áreas naturales y expansión de la ganadería extensiva (Hoogesteijn, 2003; Inskip \& Zimmerman, 2009). Por lo anterior, los objetivos de la presente investigación fueron: a) determinar la estructura y densidad poblacional del jaguar, b) analizar los patrones de actividad del jaguar y su traslape temporal con el puma (Puma concolor), c) determinar las abundancias relativas de presas potenciales, d) evaluar los daños por depredación de animales domésticos, e) implementar recomendaciones de manejo para la conservación del jaguar que minimicen el conflicto humanos-depredadores en la Reserva de la Biosfera "El Cielo", Tamaulipas, México.

\section{MATERIALES Y MÉTODOS}

Área de estudio: El municipio de Gómez Farías se ubica en la porción media del Estado de Tamaulipas, sobre la cuenca hidrológica del río Guayalejo (2242’32'N - 0³0'43”'W). Limita al norte con el municipio de Llera; al sur con el de Mante; al este con el de Xicotencatl y al oeste con el de Ocampo. Su extensión territorial es de $1445.3 \mathrm{~km}^{2}$ y está situado a 350 msnm (Medrano, 2005; SánchezRamos et al., 2005).

Con respecto a su orografía, la porción oriental del Municipio de Gómez Farías corresponde a la llanura costera del Golfo y está formada por terrenos sensiblemente planos. Así mismo, las estribaciones presentes corresponden a la Sierra Madre Oriental (Sierra de Cucharas) de terrenos sinuosos cuya altitud varía entre los 1500 y 1900 msnm. La vegetación predominante del área es bosque tropical caducifolio, bosque tropical subperennifolio, bosques de coníferas, chaparral, bosque mesófilo de montaña, vegetación secundaria y áreas de cultivo (Medrano, 2005).

La precipitación y temperatura media anual varían de 95.2 a $1028.7 \mathrm{~mm}$ y de 14 a $25.2{ }^{\circ} \mathrm{C}$, respectivamente (Medrano, 2005). El periodo seco únicamente abarca dos meses (marzo-abril). El periodo de lluvia abarca diez meses (mayo-febrero), ya que se combina las lluvias con la temporada de nortes (Medrano, 2005). Los suelos son de tipo litosoles, vertisol, arcillosos y regosoles (Medrano, 2005). Las corrientes superficiales más importantes son el río Sabinas y el río Frío, las cuales atraviesan el territorio en diferentes direcciones y se unen en el municipio antes de verter sus aguas en el Guayalejo o Tamesí (Medrano, 2005) (Fig. 1).

En la cabecera municipal viven cerca de 2000 personas. La mayoría de los jóvenes y adultos se dedican al corte de palma camedor (Chamaedorea sp.), al cultivo del nopal y mango, así como al ecoturismo. De forma temporal cultivan maíz, frijol y calabaza. Los principales animales domésticos en el área son: gallinas, cerdos, borregos, burros, mulas, perros y gatos (CONANP, 2013). 


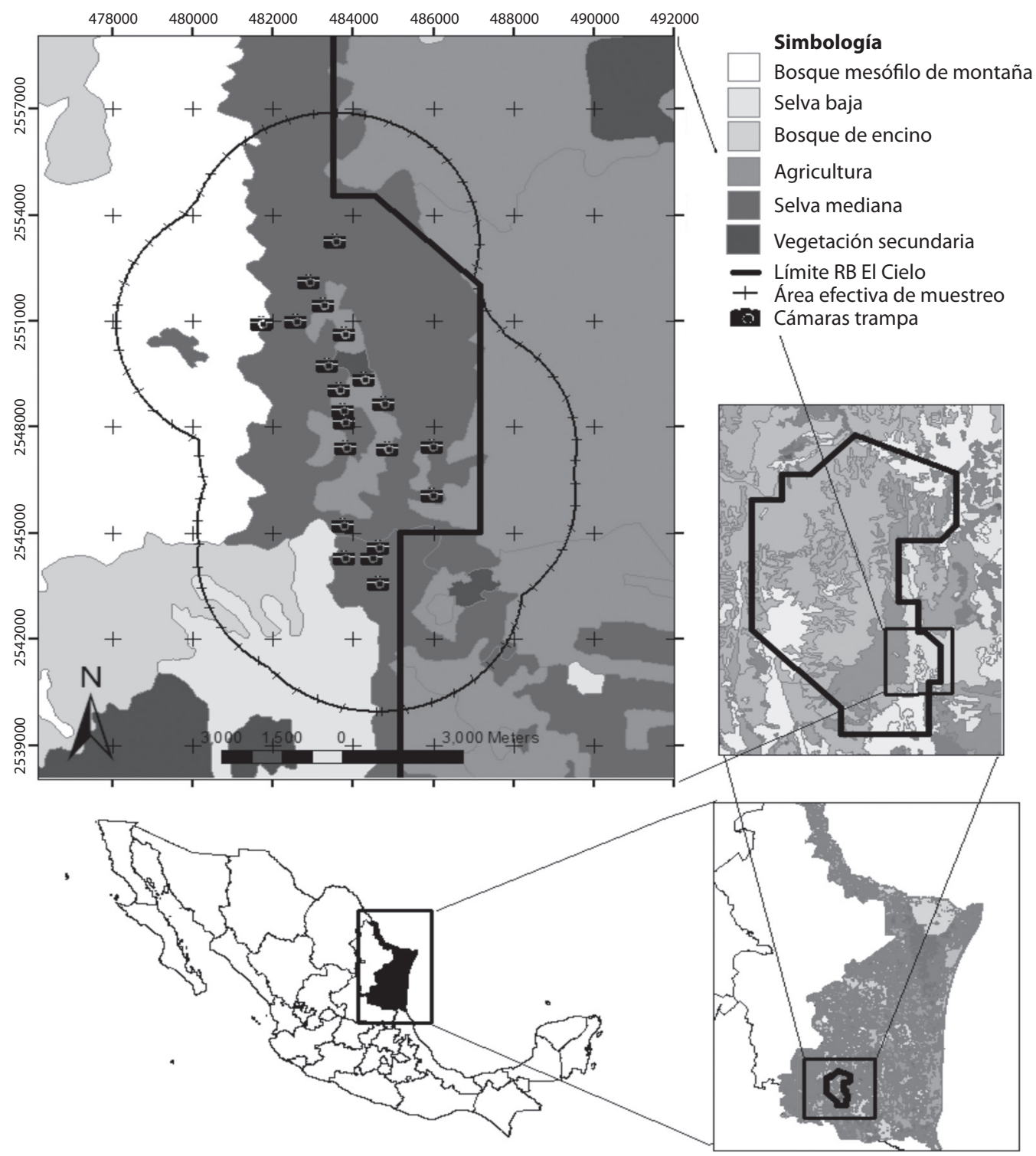

Fig. 1. Sitio de estudio y distribución de las cámaras trampas en la cabecera municipal de Gómez Farías, en la Reserva de la Biosfera El Cielo, Tamaulipas, México.

Fig. 1. Study site and camera trap distribution in the Township of Gómez Farias in El Cielo Biosphere Reserve, Tamaulipas, Mexico.

Diseño de muestreo: Se realizó un muestreo sistemático mediante cámaras trampa entre abril 2013 y abril 2014. Se colocaron 44 cámaras trampa en 22 estaciones dobles (Chávez et al., 2007; Chávez et al., 2013). El esfuerzo total de muestreo se obtuvo multiplicando el número total de estaciones dobles por el total de días de muestreo (Medellín et al., 2006).

Las estaciones de muestreo se instalaron en veredas naturales, bordes de montañas, cañadas, arroyos secos y caminos establecidos en los alrededores de la cabecera municipal de 
Gómez Farías, en lugares donde se permitió el acceso por parte de los propietarios de los terrenos, espaciadas una de otra a una distancia de $1 \mathrm{~km}$ para no dejar áreas sin muestrear, y asumiendo que esta distancia es lo suficientemente grande para lograr la independencia estadística entre las estaciones de trampeo (Chávez et al., 2013). En cada estación se instalaron dos cámaras trampa marca BUSHNELL HD® modelo 119437 con sensor de movimiento, a una altura no mayor de $40 \mathrm{~cm}$ del nivel del suelo, dependiendo de la topografía e inclinación del área de muestreo. El circuito de estas cámaras fue programado para captar video y permanecer activo durante 24 horas. La posición de cada una de ellas fue georreferenciada con un sistema de posicionamiento global (GPS) modelo Garmin etrex®. Durante el muestreo, las cámaras fueron revisadas al menos una vez al mes; en cada video detectado se imprimió la hora y fecha (Fig. 1).

\section{Estructura y densidad poblacional del} jaguar: La identificación de los jaguares se basó en el patrón de manchas en el cuerpo (Karanth \& Nichols, 1998). Adicionalmente se identificó el sexo de los individuos y la clase de edad (crías, jóvenes o adultos). Se elaboró un catálogo de foto-identificación de los jaguares que pudieron ser identificados. Los registros obtenidos se utilizaron para construir una matriz mensual de cada individuo.

Se utilizó la técnica de captura-marcarecaptura para estimar el tamaño poblacional de la especie mediante el programa CAPTURE (http://www.mbr.-pwrc.usgs.gov/software. html) (Zamora, 2012; Chávez et al., 2013). Este programa genera estimaciones de tamaño poblacional a partir del número de individuos capturados y la proporción de recapturas. Este programa es válido solo para aquellos animales que como los felinos manchados, pueden diferenciarse a nivel individual (Karanth \& Nichols, 1998). Se construyó una matriz de datos con el historial de captura de cada individuo fotografiado (Zamora, 2012; Chávez et al., 2013). Cada fila de la matriz describió la historia de un individuo durante el muestreo, de modo que se reflejó mediante 1 la captura y 0 la no captura de ese individuo durante el periodo de muestreo (Karanth \& Nichols, 1998).

El programa CAPTURE calculó estimaciones de abundancia bajo siete modelos que difieren según la fuente de variación en la probabilidad de captura, dando un valor de 1.00 al modelo que mejor se ajuste a los datos, pero la selección final del modelo es relativa, por lo que existe un espacio para la interpretación (White, Anderson, Burnham, \& Otis, 1982). Sin embargo, se recomienda seleccionar los modelos con un valor $\geq 0.90$, es decir, con un ajuste significativo (Otis, Burnham, White, \& Anderson, 1978).

Para la estimación de la densidad se empleó la siguiente formula:

$$
\mathrm{D}=\mathrm{N} / \mathrm{A}
$$

$\mathrm{N}=$ abundancia o tamaño poblacional calculado por CAPTURE.

$\mathrm{A}=$ área efectiva de muestreo en $\mathrm{km}^{2}$, incluyendo el área adicional.

Para el cálculo del área efectiva de muestreo se tomó en cuenta una franja externa, la cual se deriva para que la distribución de los animales capturados en las cámaras-trampa no se limite solamente al perímetro de las mismas. Para calcular este buffer, se utilizaron las máximas distancias recorridas por cada individuo, es decir, para los individuos recapturados se midió la distancia máxima entre las estaciones donde fueron capturados, con estos datos se obtuvo el promedio de las distancias máximas recorridas (Mean Maximum Distance MovedMMDM), posteriormente se generó un Polígono Mínimo Convexo, y a este se le agregó el buffer construido con el MMDM, con lo que se obtuvo el área efectiva de muestreo (GonzálezMaya, Finegan, Schipper, \& Casanoves, 2008; Maffei, Noss, Silver, \& Kelly, 2011; Chávez et al., 2013) (Cuadro 1, Fig. 1).

Patrón de actividad del jaguar y traslape temporal con el puma (P. concolor): Este se determinó con al menos 10 registros videográficos independientes de las especies (Maffei, 
CUADRO 1

Historial del registro mensual de jaguares y Máxima Distancia Recorrida (MMDM) a la que se registraron mediante $\mathrm{f}$ oto-trampeo en la cabecera municipal de Gómez Farías, Tamaulipas, México, (periodo: abril 2013 - abril 2014)

TABLE 1

Individual jaguar monthly record and Mean Maximum Distance Moved (MMDM) from camera-trapping in the Township of Gomez Farias, Tamaulipas, Mexico, from April 2013 to April 2014

\begin{tabular}{|c|c|c|c|c|c|c|c|c|c|c|c|c|c|c|c|c|c|}
\hline \multirow{2}{*}{$\begin{array}{l}\quad \text { Especie } \\
\text { Carnivora } \\
\text { Felidae }\end{array}$} & \multirow[b]{2}{*}{$\begin{array}{l}\text { Nombre de } \\
\text { Identificación }\end{array}$} & \multirow[b]{2}{*}{ Sexo } & \multirow[b]{2}{*}{ Edad } & \multicolumn{13}{|c|}{ Historial de Registro Mensual 2013 - 2014} & \multirow{2}{*}{$\begin{array}{l}\text { Máxima } \\
\text { Distancia } \\
\text { Recorrida } \\
\text { (MMDM) }\end{array}$} \\
\hline & & & & Abr. & May. & Jun. & Jul. & Ago. & Sep. & Oct. & Nov. & Dic. & Ene. & Feb. & Mar. & Abr. & \\
\hline \multirow[t]{9}{*}{ Panthera onca } & Misteriosa & $\mathrm{H}$ & A & 1 & 1 & 1 & 1 & 1 & 1 & 1 & 1 & 1 & 1 & 1 & 1 & 1 & 6.8 \\
\hline & Niña & $\mathrm{H}$ & A & 1 & 0 & 1 & 1 & 1 & 0 & 1 & 0 & 0 & 0 & 0 & 0 & 0 & 4.7 \\
\hline & Tambo & M & A & 1 & 1 & 0 & 1 & 1 & 0 & 0 & 0 & 0 & 1 & 0 & 1 & 1 & 5.8 \\
\hline & Ojitos & $\mathrm{H}$ & A & 1 & 1 & 1 & 1 & 1 & 0 & 1 & 0 & 0 & 0 & 0 & 0 & 0 & 8.7 \\
\hline & Toña & $\mathrm{H}$ & A & 0 & 1 & 1 & 1 & 1 & 1 & 1 & 1 & 1 & 1 & 1 & 1 & 1 & 7.9 \\
\hline & Princesa & $\mathrm{H}$ & $\mathrm{J}$ & 0 & 1 & 0 & 1 & 1 & 0 & 1 & 0 & 0 & 1 & 1 & 0 & 0 & 6.7 \\
\hline & Sanote & M & A & 0 & 0 & 0 & 0 & 0 & 0 & 1 & 0 & 0 & 0 & 0 & 1 & 0 & 8.7 \\
\hline & Perdido & M & $\mathrm{J}$ & 0 & 0 & 0 & 0 & 0 & 0 & 0 & 1 & 0 & 1 & 0 & 0 & 0 & 8.7 \\
\hline & PROMEDIO & & & & & & & & & & & & & & & & 7.25 \\
\hline
\end{tabular}

*Abreviaturas: $\mathrm{H}=$ Hembra, $\mathrm{M}=$ Macho, $\mathrm{A}=$ Adulto, $\mathrm{J}=$ Juvenil, $\mathrm{C}=$ Cría.

Cuellar, \& Noss, 2002; Monroy-Vilchis, Zarco-González, Rodriguez-Soto, Soria-Díaz, \& Urios, 2011). Los registros obtenidos se ordenaron por intervalos de una hora y se agruparon en tres periodos: a) diurnos (08:00-17:00hr), b) nocturnos (20:00-05:00hr), y c) crepusculares, cuando se obtuvieron al amanecer (05:0008:00hr) o al atardecer (17:00-20:00hr). Se realizó una prueba de Chi cuadrado (Prueba de $\mathrm{G}$ ajustada para continuidad con la corrección de Williams) para evaluar si la proporción de los registros en los tres periodos de tiempo fueron iguales para ambas especies de felinos (Ott, 1988).

\section{Abundancia relativa de presas poten-} ciales: Para obtener el índice de abundancia relativa (IAR) de presas potenciales del jaguar se utilizó la siguiente fórmula (Maffei et al., 2002; Sanderson 2004; Azuara, 2005; Jenks et al., 2011; Zamora, 2012):

$$
\mathrm{IAR}=[\mathrm{C} / \mathrm{EM}]^{*}(1000 \text { días trampa })
$$

Donde: C es el número de eventos videográficos (independientes); EM es el esfuerzo de muestreo (medido como número de cámaras multiplicado por los días de monitoreo) estacional o total y 1000 días - trampa (unidad estándar).

Se consideraron como registros independientes los siguientes casos: a) videos consecutivos de diferentes individuos y/o especies, y b) videos consecutivos de la misma especie separados por $60 \mathrm{~min}$ (este criterio fue aplicado cuando no era claro si una serie de videos correspondía a la misma presa, de modo que los videos tomados antes de $60 \mathrm{~min}$ se consideraron como un solo registro) (Maffei et al., 2002; Sanderson, 2004; Azuara, 2005; Jenks et al., 2011; Zamora, 2012).

Entrevistas: Se hicieron conversaciones formales, informales y aplicación de cuestionarios semi-estructurados sobre avistamientos de jaguares a ganaderos, agricultores y pobladores de la región (Hernández-Sampieri, 1991; Furze, Lacy, \& Birckhead, 1996; López-Romo, 1998). El criterio de elegibilidad de los actores sociales fue que posean animales domésticos y en su caso, que hayan sufrido daños por jaguar. Las conversaciones fueron dirigidas a pobladores 
de los municipios de Gómez Farías y Llera en la Reserva de la Biosfera "El Cielo". El preguntar por otras especies de depredadores facilitó el flujo de información debido a que los entrevistados no se sintieron presionados por responder sólo asuntos relacionados con el jaguar, el cual es prohibido cazar.
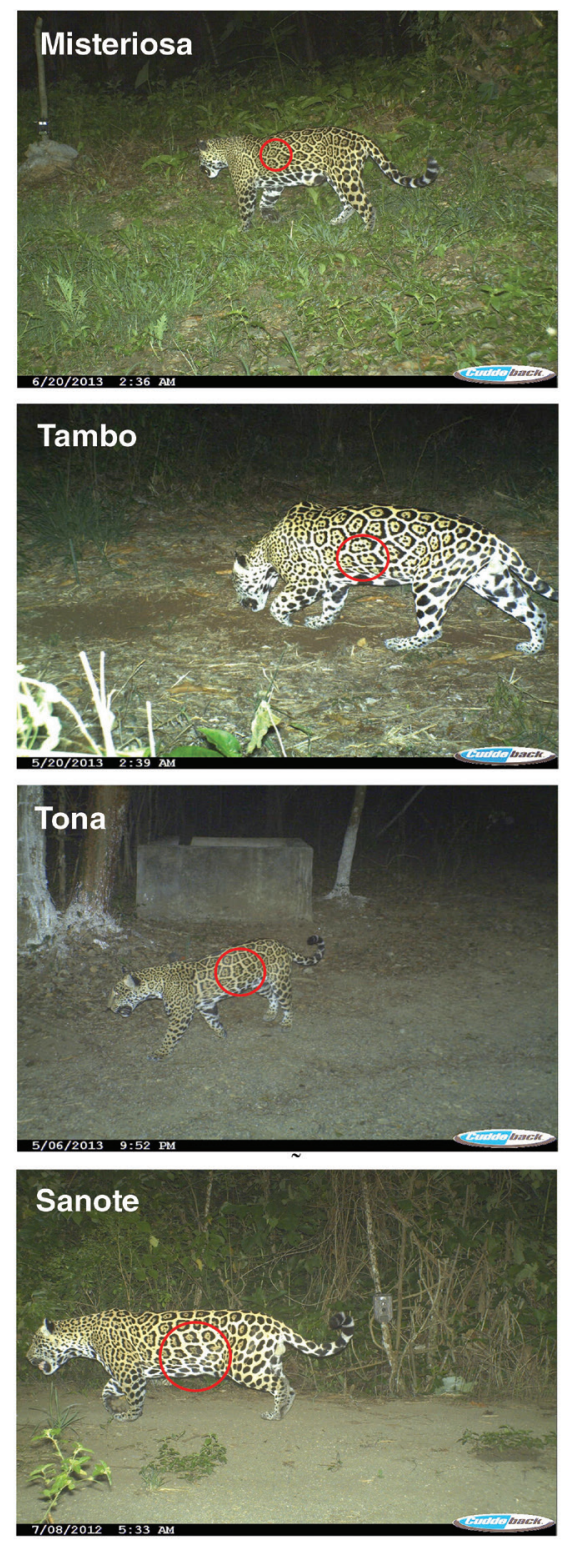

\section{RESULTADOS}

Estructura y densidad poblacional: Se identificaron ocho individuos de jaguar, incluyendo cuatro hembras adultas, una hembra juvenil, dos machos adultos y un macho juvenil (Fig. 2, Cuadro 1).
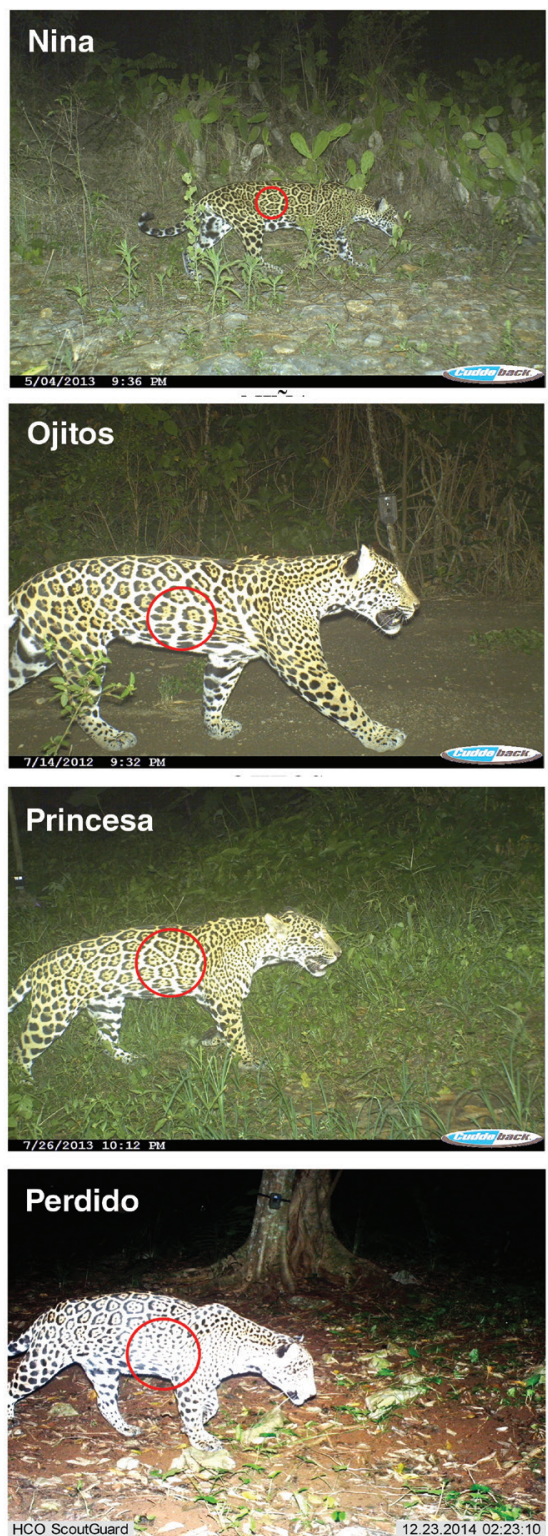

Fig. 2. Jaguares con su patrón de manchas, lo cual permite su identificación en la cabecera municipal de Gómez Farías, en la Reserva de la Biosfera El Cielo, Tamaulipas, México.

Fig. 2. Jaguar with spots pattern, allowing identification in the Township of Gómez Farias in El Cielo Biosphere Reserve, Tamaulipas, Mexico. 
Se estimó una población de 8.0 individuos $(\mathrm{ES}=1.3)$, con un intervalo de confianza del 95 $\%$ y una probabilidad de captura 0.50 . La prueba de población cerrada resultó significativa $(Z=-1.9, \mathrm{P}=0.02)$, sugiriendo una población abierta. El modelo de heterogeneidad $\mathrm{M}(\mathrm{h})$ fue el más indicado (criterio de 0.9) por lo que se observó variabilidad en la probabilidad de captura entre los jaguares.

Para estimar la densidad de jaguares, se elaboró un Polígono Mínimo Convexo entre las cámaras, y se estimó un área de 21.64 km² cubiertos por estas. Posteriormente se calculó el Promedio de la Distancia Máxima Recorrida (MMDM) para todos los individuos recapturados, obteniendo un promedio de $7.25 \mathrm{~km}$ recorridos. A partir de esto se utilizó un radio de $3.62 \mathrm{~km}$ para la construcción del buffer, obteniendo un área efectiva de muestreo de $134.561 \mathrm{~km}^{2}$. Utilizando estos datos se estimó una densidad de $5.9 \pm 1.3$ ind./100 km².

Patrón de actividad del jaguar y traslape temporal con el puma ( $P$. concolor): Con respecto a los grandes felinos existe un traslape en los patrones de actividad mostrados por ambas especies, ya que tanto el jaguar $(P$. onca) como el puma ( $P$. concolor) presentan un comportamiento nocturno-crepuscular, con registros de actividades entre las 18:00 a las 8:00 (Fig. 3). La prueba de Chi cuadrada, para evaluar si la proporción de los registros en los tres periodos de tiempo fueron iguales para ambas especies de felinos, no fue significativa $(\mathrm{G}=4.77 ; \mathrm{P}=0.21)$.

\section{Abundancia relativa de presas potencia-}

les: Se estimó un esfuerzo total de muestreo de 8580 días/trampa. Las especies de mamíferos más abundantes en las estaciones de trampeo fueron: Cuniculus paca (IAR $=9.32, \mathrm{n}=$ 80), Mazama temama (IAR $=8.62, \mathrm{n}=74)$, Odocoileus virginianus $(\mathrm{IAR}=5.01, \mathrm{n}=43$ ) y Didelphis virginiana (IAR $=2.33, \mathrm{n}=20$ ). Mientras que Mephitis macroura y Procyon lotor (IAR=0.23, $\mathrm{n}=1$ ) fueron las especies menos abundantes. Para el caso de las aves, Crax rubra fue más abundante (IAR $=25.87$, $\mathrm{n}=222$ ) que Penelope purpurascens (IAR $=$ $0.46, \mathrm{n}=4$ ) (Cuadro 2).

Entrevistas: Se aplicaron 136 encuestas a los residentes de las comunidades rurales de la "Cabecera Municipal de Gómez

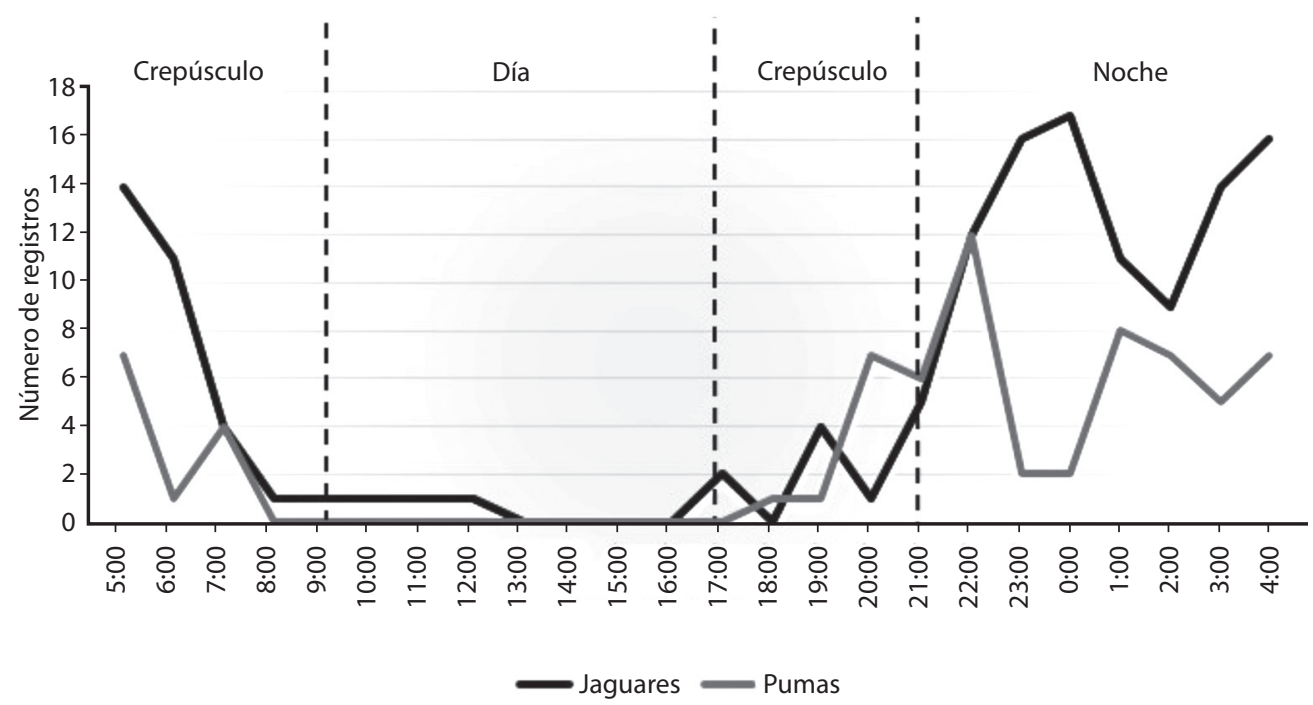

Fig. 3. Patrones de actividad del jaguar (Panthera onca) y puma (Puma concolor) en la cabecera municipal de Gómez Farías, en la Reserva de la Biosfera El Cielo, Tamaulipas, México.

Fig. 3. Activity patterns of jaguar (Panthera onca) and cougar (Puma concolor) in the Township of Gómez Farias in El Cielo Biosphere Reserve, Tamaulipas, Mexico. 
CUADRO 2

Presas potenciales reportadas para jaguar (Panthera onca) en la Reserva de la Biosfera El Cielo, Tamaulipas, México, entre abril 2013 y abril 2014, Índices de abundancia relativa (IAR) y patrones de actividad de las especies registradas

TABLE 2

Number of potential prey of jaguars (Panthera onca) photographic records and their relative abundance in El Cielo Biosphere Reserve, Tamaulipas, Mexico, between April 2013 and April 2014

\begin{tabular}{cllccl}
\multirow{2}{*}{ Clase } & Familia & \multicolumn{1}{c}{ Especie } & $\begin{array}{c}\text { Núm. Total de } \\
\text { Fotografias }\end{array}$ & IAR Total & \multicolumn{1}{c}{ Patrones de Actividad } \\
\cline { 3 - 3 } & Didelphidae & Didelphis virginiana & 20 & 2.33 & Nocturno/Crepuscular \\
& Mephitidae & Mephitis macroura & 1 & 0.23 & Nocturno \\
& Procyonidae & Nasua narica & 9 & 1.04 & Crepuscular/Diurno \\
& & Procyon lotor & 1 & 0.23 & Crepuscular \\
& Cervidae & Mazama temama & 74 & 8.62 & Diurno/Nocturno/Crepuscular \\
& & Odocoileus virginianus & 43 & 5.01 & Diurno/Nocturno/Crepuscular \\
& Tayassuidae & Dicotyles angulatus & 17 & 1.98 & Crepuscular/Diurno \\
& Cuniculidae & Cuniculus paca & 80 & 9.32 & Nocturno/Crepuscular \\
& Leporidae & Sylvilagus floridanus & 26 & 3.03 & Nocturno/Crepuscular \\
& Cracidae & Crax rubra & 222 & 25.87 & Crepuscular/Diurno \\
& & Penelope purpurascens & 4 & 0.46 & Crepuscular/Diurno \\
\hline
\end{tabular}

Farías", "Nuevo Pensar del Campesino", "Ojo de Agua", "El Azteca", en el municipio de Gómez Farías y "El Peñon" en el Municipio de Llera, en el Estado de Tamaulipas. El rango de edad de los entrevistados fue de 18-60 años (37\% hombres y $63 \%$ mujeres). La mayoría de las entrevistas se realizaron en la comunidad de Gómez Farías y Nuevo Pensar del Campesino, debido a que son las comunidades con el mayor número de habitantes y donde se han reportado más conflictos con el jaguar en los últimos años. Las comunidades de Gómez Farías y El Peñón reportaron la incidencia más alta de casas que han sufrido ataques por jaguar con $60 \%$ y $61 \%$, respectivamente, mientras que las comunidades de El Azteca y El Ojo de Agua reportaron una menor proporción (19\% y $14 \%$ respectivamente). Al agrupar las cuatro comunidades resulta que el $40 \%$ de las casas encuestadas no cuentan con algún tipo de protección para sus animales de traspatio (e.g. cerco o barda perimetral, jaulas, corrales o gallineros). Por otro lado, en los domicilios que si protegen a sus animales o toman algún tipo de medida preventiva, la infraestructura es muy básica, por lo que no garantizan la seguridad de los mismos.
En cuanto al tipo de animales domésticos depredados por jaguar, las comunidades de Gómez Farías-Nuevo Pensar y Ojo de Agua reportaron ataques principalmente en aves de corral con un $72 \%$ y $53 \%$, respectivamente, mientras que en El Peñón Llera fueron los caninos (71 \%), y en El Azteca los ovinos (60\%). Después de las aves de corral, los perros, los gatos y los cerdos fueron los animales de traspatio más depredados por jaguares $(\mathrm{n}=107)$ (Cuadro 3).

\section{DISCUSIÓN}

Existen diferentes variables que pueden afectar y limitar la estimación de densidades por medio de cámaras trampa (Silver et al., 2004), dentro de estas se incluyen la disponibilidad de senderos, caminos o rutas de uso por el jaguar (Maffei et al., 2002; Silver et al., 2004), el área de muestreo, la accesibilidad y logística para el mantenimiento y permanente funcionamiento de los equipos, niveles de impactos humanos, así como vegetación y topografía (Silver et al., 2004). Todas estas variables pueden influir en las diferentes frecuencias de captura entre áreas de estudio. Otras restricciones 


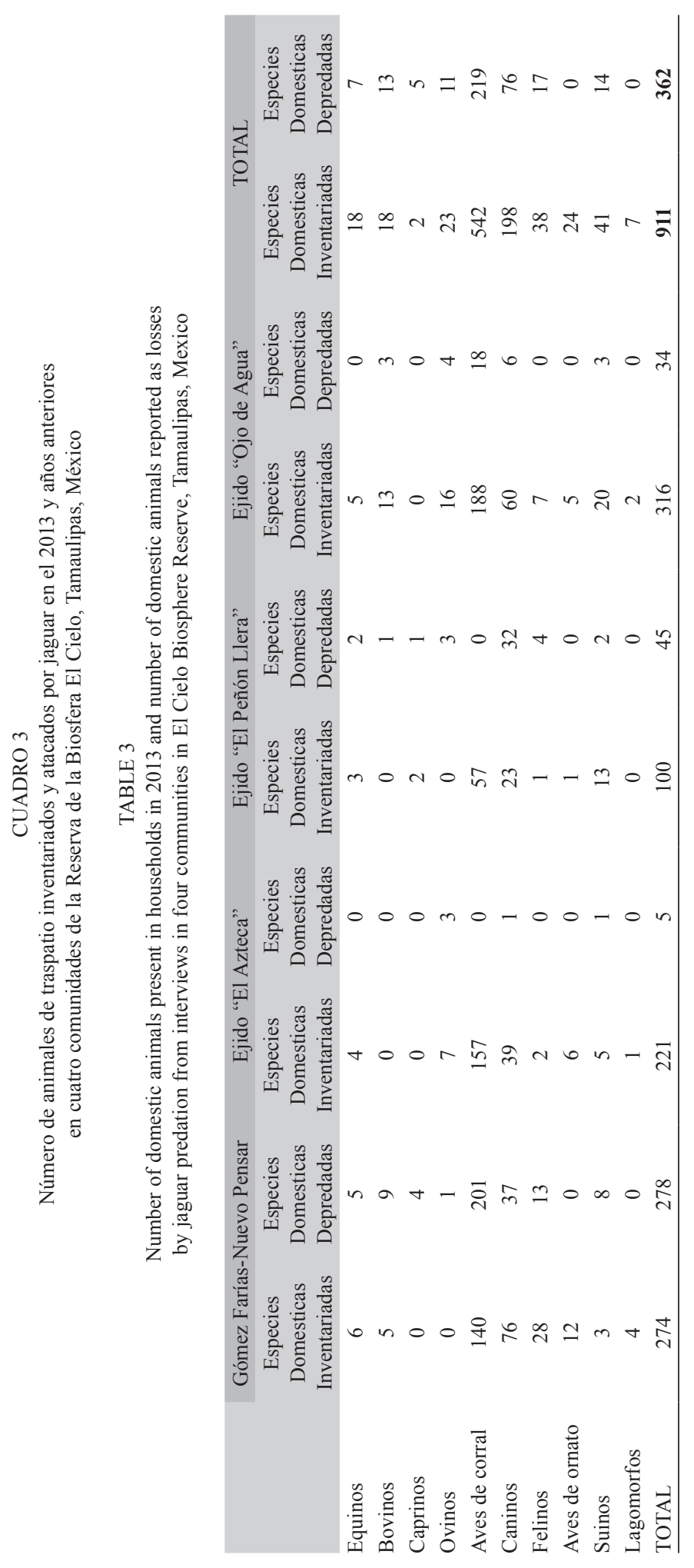


en el uso de cámaras trampa, como el diseño para cumplir los supuestos de población cerrada, representan restricciones para la estimación de densidad, donde el periodo de muestreo se debe restringir a periodos cortos de tiempo, debido a esto es posible que diferentes individuos usen la misma área en diferentes periodos (Wallace, Gómez, Ayala, \& Espinoza, 2003; Maffei, Barrientos, Mendoza, Ity \& Noss, 2003), y por ende, no todos los individuos sean capturados en el periodo de muestreo, afectando la estimación (Maffei et al., 2011).

Con respecto a la densidad estimada, la Península de Yucatán está entre las regiones mejor estudiadas, con estimaciones mediante el uso de telemetría y cámaras trampa que van de los 6.1 a 6.6 ind $/ 100 \mathrm{~km}^{2}$ (Faller, Chávez, Johnson, \& Ceballos, 2007; Chávez, 2010). Por otro lado, mediante el uso de cámaras trampas y con periodos de muestreo cortos (dos meses), De la Torre (2008) y Núñez-Pérez (2011) estiman 5.4 ind./100 km² para la Selva Lacandona en el Estado de Chiapas y para la Reserva de la Biosfera de Chamela-Cuixmala en la Costa de Jalisco respectivamente. Finalmente, RosasRosas (2006); también mediante el uso de cámaras trampa en Sonora, reporta la densidad más baja para el país; 1.0 ind./100 km². Así mismo, a nivel internacional, las estimaciones de la densidad poblacional en jaguar oscilan entre 2.0 hasta 11.4 ind. $/ 100 \mathrm{~km}^{2}$ (Maffei et al., 2011). Por lo que posiblemente la alta densidad obtenida para la Reserva de la Biosfera "El Cielo"; $5.9 \pm 1.3$ ind. $/ 100 \mathrm{~km}^{2}$, está determinada por el largo periodo de muestreo, a la presencia de una población abierta, misma que permite la emigración y migración latitudinal y longitudinal en esta área natural, lo que ha permitido su permanencia (Silver et al., 2004; Maffei et al., 2002; González-Maya et al., 2008) (Cuadro 4).

En cuanto al modelo seleccionado $\mathrm{M}(\mathrm{h})$, Karanth y Nichols (2002) recomiendan seguir este modelo, ya que es el que más se ajusta al comportamiento de los grandes felinos solitarios y territoriales como el jaguar. $\mathrm{Al}$ respecto, Ceballos, Chávez, Rivera y Manterola (2002) documentaron que los machos de jaguar son territoriales y tienen áreas de actividad mayores que las hembras en la Reserva de la Biosfera de Calakmul, México. Algo muy similar registramos, ya que uno de los jaguares machos adultos residentes registrados; "Sanote", fue fotografiado también en el ejido El Julilo por los habitantes que realizan monitoreo comunitario a $40 \mathrm{~km}$ en línea recta de nuestro sitio de estudio, así como por una hembra joven; "Princesa", quien también fue registrada a $25 \mathrm{~km}$ en el ejido La Joya de Manantiales. Por lo que muy posiblemente el cálculo del área efectiva de muestreo hecha en el presente estudio subestimo la Distancia Máxima Recorrida (MMDM) que realmente recorren estos depredadores, por lo que la densidad real del jaguar podría ser menor para esta región.

En lo concerniente a los patrones de actividad y al traslape temporal con el puma, los resultados muestran un empalme en los horarios de actividad nocturno-crepuscular para ambos depredadores. Al respecto, se ha reportado que en Belice (Rabinowitz \& Nottingham, 1986), Perú (Emmons, 1987) y Paraguay (Taber, Novaro, Neris, \& Colman, 1997) el jaguar es simpátrico con el puma ( $P$. concolor), y que dividen el tamaño y tipo de presa para reducir la competencia en las áreas en las que se traslapan. Al respecto, en las sabanas del oeste de Venezuela, se ha reportado que el puma tomará una amplia variedad de presas más pequeñas (Farell, Roman, \& Sunquist, 2000; Scognamillo, Maxit, Sunquist, \& Polisar, 2003), mientras que el jaguar tiende a tomar presas más grandes (Rabinowitz \& Nottingham, 1986; Iriarte, Franklin, Johnson, \& Redford, 1990; Jaksic, Greene, \& Yáñez, 1981) en áreas donde las presas son escasas y por ende sus encuentros son inevitables. Este comportamiento, además de las constantes actividades antropogénicas de los pobladores, y la presencia de animales domésticos en el Municipio de Gómez Farías en la Reserva de la Biosfera El Cielo, posiblemente sea la respuesta al traslape y convivencia de ambos depredadores, ya que el jaguar tomará lo que encuentra generalmente en proporción a su disponibilidad 


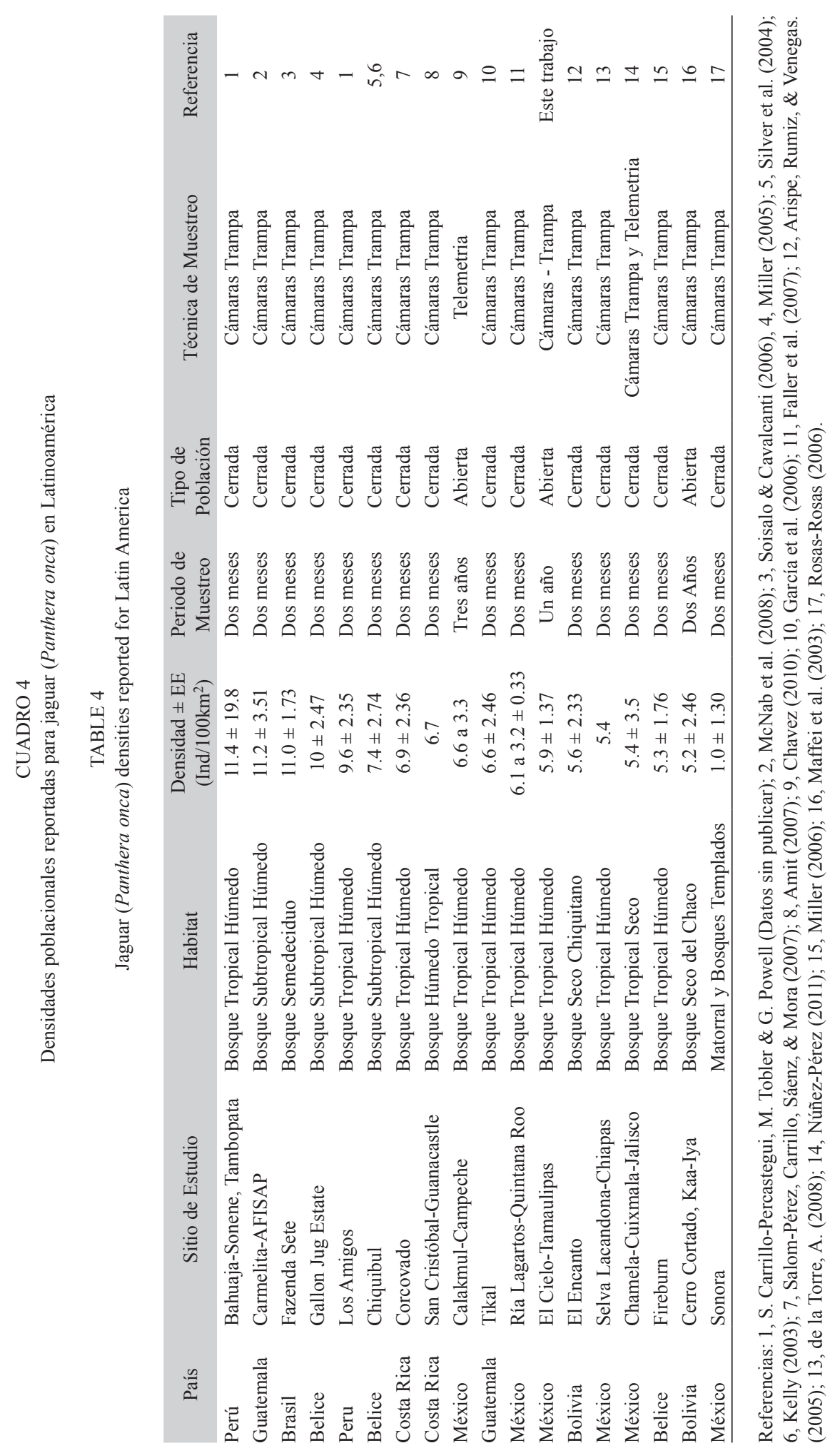


y a que se ha adaptado a consumir otro tipo de presas alternativas (Emmons, 1987).

En el presente estudio, y con base en la distribución geográfica de las especies, se ha considerado a Cuniculus paca, Mazama temama, Odocoileus virginianus, Didelphis virginiana, Dicotyles angulatus, Procyon lotor y Crax rubra como presas potenciales del jaguar similar a lo reportado en otras regiones de México (Núñez, Miller, \& Lindzey, 2002; Amín, 2004; Rosas-Rosas, Bender, \& Valdez, 2008). No obstante, los perros, gatos y cerdos domésticos también están siendo parte de su dieta. Estudiar las presas es un factor importante para conservar al jaguar en esta región, por lo que recomendamos seguir monitoreando la abundancia relativa de las presas potenciales en diferentes mosaicos de vegetación y gradientes altitudinales, para ver tendencias con base en disponibilidad, abundancia y distribución. Puesto que mientras para algunas presas los índices de abundancia están en el rango o por encima de otras regiones del Neotrópico, para otras están por abajo (Lira-Torres \& BrionesSalas, 2012). Con esto se abre la posibilidad de nuevos estudios para inferir sobre lo que pasa con estas especies a través del tiempo y su dinámica poblacional.

En relación a las entrevistas y a la frecuencia en la depredación de animales domésticos en el área de estudio, es importante comentar que posiblemente los ataques continuarán, mientras se siga teniendo la infraestructura actual en las comunidades para resguardar sus animales, y no se implementen medidas preventivas y disuasivas que disminuyan estos ataques. Al respecto, autores como AmadorAlcalá, Naranjo y Jiménez-Ferret (2013) han atribuido estos ataques por parte de los felinos silvestres a: 1) felinos incapacitados para cazar presas naturales (e.g. individuos viejos, juveniles inexpertos o animales heridos o con dientes fracturados), 2) la disminución de presas naturales por efecto de la cacería no controlada, y 3 ) falta de cuidado en el alojamiento de los animales domésticos. Este último punto algo común detectado y registrado durante las entrevistas.
El jaguar es una especie oportunista y versátil, a falta de presas naturales rápidamente hace uso del recurso disponible (Núñez, 2007). Por lo que para reducir este conflicto en las comunidades que conforman esta reserva de la biosfera recomendamos: a) educación ambiental: Informar a las comunidades y pobladores del papel en el ecosistema del jaguar y las consecuencias de su erradicación. b) disponer convenientemente de los cadáveres de animales domésticos muertos por otras causas (mordidas de serpiente $y / o$ enfermedades, entre otros). c) promover el pago del seguro ganadero por el gobierno federal. d) control de los animales domésticos mediante la implementación de corrales con buena estructura. e) implementar los comités de vigilancias comunitarias y atender los casos de depredación por jaguares. f) implementación de medidas preventivas y de disuasión. Las medidas preventivas pueden incluir cercos eléctricos, y las medidas de disuasión van orientadas a mostrar al depredador que no es bien recibido en las comunidades. Estos métodos incluyen el uso de perros de guardia, perros de disuasión, repelentes sistémicos (e.g. Thiabendazole), alarmas con sensor de movimiento y luces estroboscópicas, entre otros.

Finalmente, el jaguar es considerado una especie focal por su alta sensibilidad a la cacería, a la fragmentación y pérdida de hábitat, a la disminución de sus presas potenciales, y a que utiliza una gran extensión de terreno con diferentes tipos de hábitat. Por lo tanto, al proteger esta especie, se garantiza la conservación de una gran cantidad de especies con las que coexiste (Miller \& Rabinowitz, 2002; Drury, Omewood, \& Randall, 2011). Por lo que la presencia del jaguar en la Reserva de la Biosfera "El Cielo", resulta un buen indicador del estado de conservación de esta área natural en México.

\section{AGRADECIMIENTOS}

Agradecemos el apoyo financiero de la Comisión Nacional de Áreas Naturales Protegidas (CONANP) a través del Proyecto: Conservación del jaguar y análisis de conflictos con la población humana en la RPC "El Cielo", a 
través del Programa de Conservación de Especies en Riesgo 2013. Un agradecimiento especial a Mario A. Álvarez-Lara por su aportación con los primeros registros fotográficos $\mathrm{y}$ en la identificación de los ejemplares registrados en este estudio. Un reconocimiento a Sergio Mireles-Infante y Rubén Suárez-Hernández por el apoyo técnico, administrativo y la elaboración del mapa. Agradecemos al Sistema Nacional de Investigadores (SNI) y al Programa para el Desarrollo Profesional Docente para el tipo Superior (PRODEP) por su reconocimiento y apoyo.

\section{RESUMEN}

Existe poca información acerca de la ecología del jaguar (Panthera onca) para la Reserva de la Biosfera El Cielo, en el Estado de Tamaulipas, México. Los trabajos con los que se cuenta se limitan a experiencias anecdóticas y pocas publicaciones. Los objetivos de la presente investigación fueron: a) determinar la estructura y densidad poblacional del jaguar, b) analizar sus patrones de actividad y su traslape temporal con el puma (Puma concolor), c) determinar las abundancias de presas potenciales, y d) evaluar los daños por depredación en animales domésticos en el Municipio de Gómez Farías. Entre abril 2013 y abril 2014 se colocaron cámaras trampa con un esfuerzo de muestreo de 8580 días cámaras trampa. Adicionalmente, se aplicaron 136 encuestas semi-estructuradas entre residentes de las comunidades del municipio de Gómez Farías y Llera con el objeto de recabar información sobre daños en animales domésticos por ataques de jaguar y otros carnívoros silvestres. Se identificaron ocho individuos de jaguar; cuatro hembras adultas, una hembra juvenil, dos machos adultos y un macho juvenil. La densidad estimada fue de $5.9 \pm 1.3 \mathrm{ind} / 100 \mathrm{~km}^{2}$. Con respecto a los patrones de actividad del jaguar y el puma, se encontró que existe un traslape en el que ambas especies mostraron un comportamiento nocturno-crepuscular. Las especies presa más abundantes fueron: Crax rubra, Cuniculus paca, Mazama temama, Odocoileus virginianus y Didelphis virginia$n a$, mientras que las menos abundantes fueron Mephitis macroura y Procyon lotor. Los resultados de las encuestas mostraron que después de las aves de corral, los perros, los gatos y los cerdos fueron los animales de traspatio más depredados por los jaguares $(\mathrm{n}=107)$. Este estudio representa el primer esfuerzo que aporta información sobre la ecología de la especie para la reserva, sin embargo, es evidente que se necesita incrementar el monitorio para determinar la situación actual de la población, generando estrategias adecuadas para su conservación. Finalmente, consideramos que la Reserva de la Biosfera "El Cielo" representa una unidad ecológicamente importante para las poblaciones de jaguares de la Sierra Madre Oriental, por la Vertiente del Golfo de México, ya que puede funcionar como un corredor biológico entre las poblaciones de San Luis Potosí y Nuevo León, México.

Palabras clave: conflicto depredador humanos, población de jaguar, cámaras trampa, patrones de actividad, El Cielo, Tamaulipas.

\section{REFERENCIAS}

Amador-Alcalá, S., Naranjo, E., \& Jiménez-Ferret, G. (2013). Wildlife predation on livestock and poultry: implications for predator conservation in the rainforest of south-east Mexico. Oryx, 47, 243-250.

Amín, M. (2004). Patrones de alimentación y disponibilidad de presas en jaguar (Panthera onca) y del Puma (Puma concolor) en la Reserva de la Biosfera Calakmul, Campeche. México (Tesis de Maestría). Universidad Nacional Autónoma de México, México.

Amit, R. R. (2007). Densidad de jaguares (Panthera onca), en el Sector San Cristóbal del Área de Conservación Guanacaste (Tesis de grado). Universidad Nacional, Heredia, Costa Rica.

Arispe, R., Rumiz, D. I., \& Venegas, C. (2005). Segundo censo de jaguares (Panthera onca) y otros mamiferos con trampas cámara en la Estancia San Miguelito (Technical Report \#144). Santa Cruz, Bolivia: Wildlife Conservation Society.

Azuara, S. D. (2005). Estimación de abundancia de mamiferos terrestres en un área de la Selva Lacandona, Chiapas. México. (Tesis Licenciatura). UNAM, D.F.

Barea-Azcón, J. M., Virgós, E., Ballesteros-Duperón, E., Moleón, M., \& Chirosa, M. (2007). Surveying carnivores at large spatial scales: a comparison of four broad-applied methods. Biodiversity and Conservation, 16, 1213-1230.

Briones-Salas, M., Lavariega, M., \& Lira-Torres, I. (2012). Distribución actual y potencial del Jaguar (Panthera onca) en Oaxaca, México. Revista Mexicana de Biodiversidad, 83, 246-257.

Carrera, R., Cavazos, J., Briones-Salas, M., \& Lira-Torres, I. (2016). Registro actual del jaguar Panthera onca (Carnivora: Felidae) en el Parque Nacional Cumbres de Monterrey, Nuevo León, México. Revista Mexicana de Biodiversidad, 1-12.

Caso, A. (2007). Situación del Jaguar en el Estado de Tamaulipas. In G. Ceballos, C. Chávez, R. List, \& H. Zarza (Eds.), Conservación y Manejo del Jaguar en México: Estudios de Caso y Perspectivas (pp. 19-24). Ciudad de México, México: CONABIO Alianza WWF / Telcel - Universidad Nacional Autónoma de México. 
Caso, A., López-González, C., Payan, E., Eizirik, E., de Oliveira, T., Leite-Pitman, R., Kelly, M., \& Valderrama, C. (2008). Panthera onca. The IUCN Red List of Threatened Species. Version 2014.2. http//iucnredlist. org; última consulta: 20 Enero 2015.

Ceballos, G., Chávez, C., Blanco, S., Jiménez, R., López, M., Moctezuma, O., Támez, V., \& Valdez, M. (2006). Áreas prioritarias para la conservación. In C. Chávez \& G. Ceballos (Eds.), El Jaguar Mexicano en el Siglo XXI: Situación Actual y Manejo (pp. 13-19). Ciudad de México, México: CONABIO-Alianza WWF Telcel-Universidad Nacional Autónoma de México.

Ceballos, G., Chávez, Ch., Rivera, A., \& Manterola, C. (2002). Tamaño poblacional y Conservación del jaguar en la Reserva de la Biosfera Calakmul, Campeche, México. In R. A. Medellín, C. Equihua, C. L. B. Chetkiewicz, P. G. Crawshaw, Jr., A. Rabinowitz, K. H. Redford, J. G. Robinson, J. Sanderson, \& A. B. Taber (Eds.), El Jaguar en el Nuevo Milenio (pp. 403-418). Ciudad de México, México: Fondo de Cultura Económica. Universidad Nacional Autónoma de México y Wildlife. Conservation Society.

CONANP (Comisión Nacional de Áreas Naturales Protegidas). (2009). Programa de acción para la conservación de la especie: jaguar (Panthera onca). México, D. F.: Gobiernos Federal y Secretaría de Medio Ambiente y Recursos Naturales.

CONANP (Comisión Nacional de Áreas Naturales Protegidas). (2013). Conservación del Jaguar y Análisis de Conflictos en la Población Humana en la Reserva de la Biosfera "El Cielo", Tamaulipas. (Informe Final del Programa de Conservación de Especies en Riesgo (PROCER): Jaguar). México, D.F.: Comisión Nacional de Áreas Naturales Protegidas.

Chávez, C. (2010). Ecología y Conservación del Jaguar (Panthera onca) y el Puma (Puma concolor) en la Región de Calakmul y sus Implicaciones para la Conservación de la Península de Yucatán. (Tesis de Doctorado). Universidad de Granada, España.

Chávez, C. \& Ceballos, G. (2006). El Jaguar Mexicano en el Siglo XXI: Situación Actual y Manejo. D.F. México: CONABIO-ALIANZA WWF/TELCELUniversidad Nacional Autónoma de México.

Chávez, C., \& Ceballos, G. (2014). Jaguar (Panthera onca Linnaeus, 1758). In G. Ceballos (Ed.), Mammals of Mexico (pp. 507-509).USA: Johns Hopkins University Press.

Chávez, C., Ceballos, G. Medellín, R., \& Zarza, H. (2007). Primer Censo Nacional del Jaguar. In G. Ceballos, C. Chávez, R. List, \& H. Zarza (Eds.). Conservación y Manejo del Jaguar en México: Estudios de Caso y Perspectivas (pp. 113-141). Ciudad de México, México: CONABIO-Alianza WWF/Telcel-Universidad Nacional Autónoma de México.
Chávez, C., De la Torre, A., Bárcenas, A., Medellín, R., Zarza, H., \& Ceballos, G. (2013). Manual de fototrampeo para estudios de fauna silvestre. El jaguar en México como estudio de caso. Ciudad de México, México: Alianza WWF-Telcel, Universidad Nacional Autónoma de México.

Chávez-Tovar, J., Aranda, M., \& Ceballos, G. (2006). Panthera onca. In G. Ceballos \& G. Oliva (Eds.), Los Mamíferos Silvestres de México (pp. 367-370). Ciudad de México, México: Fondo de Cultura Económica y Comisión Nacional para el Conocimiento y Uso de la Biodiversidad.

Drury, R., Omewood, K., \& Randall, S. (2011). Less is more: the potential of quantitative approaches in conservation research. Animal conservation, 14, 18-24.

Dueñas-López, G. (2013). Identificación de Corredores Biológicos Potenciales para el Jaguar (Panthera onca) en la Sierra Abra Tanchipa, San Luís Potosi y sus Límites Estatales. Montecillos, México (Tesis de Maestría). El Colegio de Postgraduados, México.

Emmons, L. (1987). Comparative feeding ecology of felids in a Neotropical rainforest. Behavioral Ecology and Sociobiology, 20, 271-283.

Faller, J. C., Chávez, Ch., Johnson, S., \& Ceballos, G. (2007). Densidad de la población de jaguares en el Noreste de la Península de Yucatán. In G. Ceballos, C. Chávez, R. List, \& H. Zarza (Eds.), Conservación y Manejo del Jaguar en México: Estudios de Caso y Perspectivas (pp. 111-121). Ciudad de México, México: CONABIO-Alianza WWF/Telcel-Universidad Nacional Autónoma de México.

Farell, L., Roman, J., \& Sunquist, M. (2000). Dietary separation of sympatric carnivores identified by molecular analysis of scats. Molecular Ecology, 9, $1583-1590$.

Furze, B., Lacy, T., \& Birckhead, J. (1996). Using methods from the social sciences. In John Wiley \& Sons (Eds.), Culture, conservation and biodiversity. The social dimension of linking local level development and conservation through protected areas. Reino Unido: West Sussex.

Galindo-Leal, C. (2009). Panthera onca. Ciudad de México, México: Universidad Autónoma Metropolitana.

García, A. R., McNab, R. B., Shoender, J. S., Radachowsky, J., Moreira, J., Estrada, C., Méndez, V., ..., \& Flores, L. (2006). Los jaguares del corazón del Parque Nacional Tikal, Petén, Guatemala. Flores, Petén, Guatemala: Asociación Balám and Wildlife Conservation Society.

Gittleman, J. L., Funk, S. M., Macdonald, D. W., \& Wayne, R. K. (2001). "Why Carnivore conservation"? In J. L. Gittleman, S. M. Funk, D .W. Macdonald, \& R. K. Wayne (Eds.), Carnivore Conservation. United Kingdom: Cambridge University Press. 
González-Maya, J., Finegan, B. G., Schipper, J., \& Casanoves, F. (2008). Densidad absoluta y conservación del jaguar y sus presas en la Región Talamanca Pacífico, Costa Rica. Serie Técnica No. 7: Apoyando los esfuerzos en el manejo y protección de la biodiversidad tropical. San José, Costa Rica: The Nature Conservancy.

Grigione, M. M., Menke, K., López-González, C., List, R., Banda, A., Carrera, J., Carrera, R., ..., \& Van Pelt, B. (2009). Identifying potential conservation areas for felids in the USA and Mexico: integrating reliable knowledge across an international border. Fauna \& Flora International, Oryx, 43(1), 78-86.

Hernández-Sampieri, R. (1991). Metodología de la investigación. México: McGraw-Hill.

Hoogesteijn, R. (2003). Manual sobre problemas de depredación causados por jaguares y pumas en hatos ganaderos. New York, USA.: Wildlife Conservation Society.

Hoogesteijn, R. \& Hoogesteijn, A. (2001). Estrategias anti depredación para fincas ganaderas en Latinoamérica: Una guía. PANTHERA. Campo Grande, Mato Grosso del Sur, Brasil: Gráfica y Editora Microart Ltda.

Inskip, C., \& Zimmerman, A. (2009). Human-felid conflict: a review of patterns and priorities worldwide. Fauna \& Flora International, Oryx, 43, 18-34.

Iriarte, J., Franklin, W., Johnson, W., \& Redford, K. (1990). Biogeographic variation of food habits and body size of the American puma. Oecologia, 85, 185-190.

Jaksic, M., Greene, H., \& Yáñez, J. (1981). The guild structure of a community of predatory vertebrates in central Chile. Oecologia, 49, 21-28.

Jenks, K. E., Chanteap, P., Damrongchainarong, K., Cutter, P., Cutter, P., Redford, T., Lynam, A. J., Howard, J., \& Leimgruber, P. (2011). Using relative abundance indices from camera-trapping to test wildlife conservation hypotheses - an example from Khao Yai National Park, Thailand. Tropical Conservation Science, 4, 113-131.

Karanth, K. U., \& Nichols, J. D. (1998). Estimation of tiger densities in India using photographic captures and recaptures. Ecology, 79, 2852-2862.

Karanth, K. U., \& Nichols, J. D. (2002). Monitoring tigers and their prey, a manual for researchers, managers and conservationist in tropical Asia. Bangalore, India: Centre for Wildlife Studies.

Kelly, M. J. (2003). Jaguar monitoring in the Chiquibul Forest, Belize. Caribbean Geography, 13(1), 19-32.

Lira-Torres, I. \& Briones-Salas, M. (2012). Abundancia relativa y patrones de actividad de los mamíferos de los Chimalapas, Oaxaca, México. Acta Zoológica Mexicana (n.s.), 28(3), 566-585.

López-Romo, H. (1998). La metodología de la encuesta. In C. J. J. Galindo (Ed.), Técnicas de investigación en sociedad, cultura y comunicación (pp. 33-73). Ciudad de México, México: Addison Wesley Longman.

López-Soto, J. H., Rosas-Rosas, O., \& Niño-Ramírez, J. A. (1997). El Jaguar (Panthera onca veraecrucis) en Nuevo León México. Revista Mexicana de Mastozoologia, 2, 126-128.

Maffei, L., Barrientos, J., Mendoza, F., Ity, E., \& Noss, A. J. (2003). Jaguar and other mammal camera trap survey Cerro II, Cerro Cortado field camp (19 $31^{\circ}$ ' 36" S, 61 $18^{\prime} 36^{\prime \prime}$ W), Kaa-Iya del Gran Chaco National Park, 28 November 2002-28 January 2003. (Technical Report \#85). Santa Cruz: Bolivia Kaa-Iya Project (Capitanía de Alto y Bajo Isoso, Wildlife Conservation Society).

Maffei, L., Cuellar, E., \& Noss, J. (2002). Uso de trampas cámara para la evaluación de mamíferos en el ecotono Chaco-Chiquitanía. Revista Boliviana de Ecología y Conservación Ambiental, 11, 55-65.

Maffei, L., Noss, A. J., Silver, S. C., \& Kelly, M. J. (2011). Abundance/Density Case Study: Jaguar in the Americas. In A. F. O'Connell, J. D. Nichols, \& K. U. Karanth (Eds.), Camera Traps in Animal Ecology (pp. 119-144). Springer.

McNab, R. B., Moreira, J., García, R., Ponce, G., Méndez, V., Córdova, M., Córdova, F., ..., \& Monzón, R. (2008). Densidad de jaguares en la Concesión Comunitaria de Carmelita y Asociación Forestal Integral San Andrés Petén, Reserva de la Biosfera Maya (Technical Report). Flores, Guatemala: Wildlife Conservation Society-Guatemala.

Medellín, R., Azuara, D., Maffei, L., Zarza, H., Bárcenas, H., Cruz, E., Legaria, R., ... \& Ávila, S. (2006). Censos y Monitoreo. In C. Chávez \& G. Ceballos (Eds.), El Jaguar Mexicano en el Siglo XXI: Situación Actual y Manejo (pp. 25-35). Ciudad de México, México: CONABIO-ALIANZA WWF TELCEL-Universidad Nacional Autónoma de México.

Medrano, F. (2005). La Vegetación. In G. Sánchez-Ramos, P. Reyes-Castillo, R. Dirzo (Eds.), Historia Natural de la Reserva de la Biosfera El Cielo, Tamaulipas, México (pp. 88-105). México: Universidad Autónoma de Tamaulipas.

Miller, C. M. (2005). Jaguar density in Gallon Jug Estate, Belize (Unpublished report). Gallon Jug, Belize: Wildlife Conservation Society.

Miller, C. M. (2006). Jaguar density in Fireburn, Belize (Unpublished report). Gallon Jug, Belize: Wildlife Conservation Society. 
Miller, B. \& Rabinowitz, A. (2002). “¿Por qué conservar al Jaguar?”. In R. A. Medellín, C. Equihua, C. Chetkiewics, A. Rabinowitz, P. Crawshaw, K. Redford, J. G. Robinson, E. Sanderson, \& A. Tabler (Eds.), El Jaguar en el Nuevo Milenio (pp. 303-315). Ciudad de México, México: Fondo de Cultura Económica. Universidad Nacional Autónoma de México y Wildlife Conservation Society.

Monroy-Vilchis, O., Zarco-González, M., Rodriguez-Soto, C., Soria-Díaz, L., \& Urios, V. (2011). Fototrampeo de mamíferos en la Sierra Nanchititla, México. Revista de Biología Tropical, 59, 373-383.

Moreira, J., García, R., McNab, R., Ponce-Santizo, G., Mérida, M., \& Ruano, G. (2009). Abundancia de Jaguares y Evaluación de Presas Asociadas al Fototrampeo en las Concesiones Comunitarias del Bloque de Melchor de Mencos, Reserva de la Biosfera Maya, Petén, Guatemala (Informe Final). Guatemala: Wildlife Conservation Society, Programa para Guatemala.

Núñez, R. (2007). Distribución y situación del jaguar en el occidente de México. In G. Ceballos, C. Chávez, R. List, \& H. Zarza (Eds.), Conservación y Manejo del Jaguar en México: Estudios de Caso y Perspectivas (pp. 25-40). Ciudad de México, México: CONABIOAlianza WWF/Telcel-Universidad Nacional Autónoma de México.

Núñez, R., Miller, B., \& Lindzey, F. (2002). Ecología del jaguar en la Reserva de la Biosfera Chamela-Cuixmala, Jalisco, México. In R. A. Medellín, C. Equihua, C. L. B. Chetkiewicz, P. G. Crawshaw, Jr., A. Rabinowitz, K. H. Redford, J. G. Robinson, J. Sanderson, \& A. B. Taber (Eds.), El Jaguar en el Nuevo Milenio (pp. 107-125). Ciudad de México, México: Fondo de Cultura Económica. Universidad Nacional Autónoma de México y Wildlife. Conservation Society.

Núñez-Pérez, R. (2011). Estimating jaguar population density using camera-traps: a comparison with radio-telemetry estimates. Journal of Zoology, 285(1), 39-45.

Otis, D. L., Burnham, K. P., White, G., C., \& Anderson, D. R. (1978). Statistical inference from capture data on closed animal populations. Wildlife Monographs, $62,1-135$.

Ott, L. (1988). An Introduction to statistical methods and data analysis (3rd ed.). Boston, Massachusetts, USA: PWS-Kent Publishing Company.

Peña-Mondragón, J. L. (2011). Daños económicos al ganado y percepciones sociales sobre el jaguar (Panthera onca veraecrucis Nelson and Goldman, 1933) en la Gran Sierra Plegada, Nuevo León, México (Tesis de maestría). Universidad Nacional Autónoma de México, México.

Rabinowitz, A. R., \& Nottingham, B. G. (1986). Ecology and behavior of the jaguar (Panthera onca) in Belize, Central America. Journal of Zoology, 210, 149-159.
Ramamoorthy, T. P., Bye, R., Lot, A., \& Fa, J. (1993). Biological diversity of Mexico: Origins and distribution. New York, USA: Oxford University Press.

Rodríguez-Soto, C., Monroy-Vilchis, O., Maiorano, L., Boitani, L., Faller, J. C., Briones-Salas, M., Núñez, R., Rosas-Rosas, O., \& Ceballos, G. (2011). Predicting potential distribution of the jaguar (Panthera onca) in Mexico: identification of priority areas for conservation. Diversity and Distributions (Diversity Distrib.), 17, 350-361.

Rosas-Rosas, O. C. (2006). Ecological status and conservation of jaguars in northeastern Sonora, Mexico (Ph.D. dissertation). New Mexico State University, Las Cruces, New Mexico.

Rosas-Rosas, O., Bender, L. C., \& Valdez, R. (2008). Jaguar and puma predation on Calves in Noreastern Sonora, Mexico. Rangeland Ecology \& Management, 61, 554-560.

Salom-Pérez, R., Carrillo, E., Sáenz, J. C., \& Mora, J. M. (2007). Critical condition of the jaguar Panthera onca in Corcovado National Park, Costa Rica. Oryx, 4l(1), 1-7.

Sánchez-Ramos, G., Reyes-Castillo, P., \& Dirzo, R. (2005). Historia Natural de la Reserva de la Biosfera El Cielo, Tamaulipas, México. México: Universidad Autónoma de Tamaulipas.

Sanderson, J. G. (2004). Protocolo para Monitoreo con Cámaras para Trampeo Fotográfico. USA: Tropical Ecology Assessment and Monitoring (TEAM) Initiative. The Center for Applied Biodiversity Science (CABS). Conservación Internacional.

Sanderson, J., Chetkiewicz, C. L. B., Medellín, R., Rabinowitz, A., Redford, K. H., Robinson, J. G., \& Taber, A. (2002). Prioridades geográficas para la conservación del jaguar. In R. A. Medellín, C. Equihua, C. L. B. Chetkiewicz, P. G. Crawshaw, Jr., A. Rabinowitz, K. H. Redford, J. G. Robinson, J. Sanderson, \& A. B. Taber (Eds.), El Jaguar en el Nuevo Milenio (pp. 601-628). Ciudad de México, México: Fondo de Cultura Económica. Universidad Nacional Autónoma de México y Wildlife. Conservation Society.

Scognamillo, D. G., Maxit, I. E., Sunquist, M., \& Polisar, J. (2003). Coexistence of jaguar (Panthera onca) and puma (Puma concolor) in a mosaic landscape in the Venezuelan llanos. Journal of the Zoological Society of London, 259, 269-279.

SECRETARÍA DE MEDIO AMBIENTE, RECURSOS NATURALES (SEMARNAT). (2010). Norma Oficial Mexicana NOM-059-ECOL-2010. Protección ambiental, especies de flora y fauna silvestres de México, categorías de riesgo y especificaciones para su inclusión, exclusión o cambio, y lista de especies en riesgo. Diario Oficial de la Federación, Jueves 30 de diciembre de 2010. 1: 1-77. 
Seymour, K. L. (1989). Panthera onca. Mammalian Species, 340, 1-9.

Silver, S., Ostro, L., Marsh, L., Maffei, L., Noss, A., Kelly, M., Wallace, R., Gomez, H., \& Ayala, G. (2004). The use of camera traps for estimating jaguar Panthera onca abundance and density using capture/recapture analysis. Oryx, 38(2), 1-7.

Soisalo, M. K., \& Cavalcanti, S. M. C. (2006). Estimating the density of a jaguar population in the Brazilian Pantanal using camera-traps and capture-recapture sampling in combination with GPS radio-telemetry. Biological Conservation, 129, 487-496.

Swank, W. G., \& Teer, J. G. (1989). Status of the jaguar Fauna \& Flora International, Oryx, 23(01), 14-21.

Taber, A., Novaro, A., Neris, N., \& Colman, F. (1997). The Food Habits of Sympatric Jaguar and Puma in the Paraguayan Chaco. Biotropica, 29(2), 204-213.

Toledo, V. M. (1988). La diversidad biológica de México. Ciencia y Desarrollo, 8, 7-16.

de la Torre, A. (2008). Research for the conservation of the jaguar (Panthera onca) in the Selva Lacandona, Chiapas, Mexico (Unpublished Report). USA: Rufford Small Grants Foundation.

Vales, G. F., Rodríguez, R., De la Maza, M., Gonzáles, C., \& Bretón, C. (2000). Áreas Naturales Protegidas de México. México: Instituto Nacional de Ecología SEMARNAP.
Vidal, R. R. (1999). Donde el Bosque es de Niebla y las Aves de Esmeralda. PRONATURA, 5, 8-19.

Wallace, R., Gómez, H., Ayala, G., \& Espinoza, F. (2003). Camera trapping for jaguar (Panthera onca) in the Tuichi valley, Bolivia. Mastozoologia Neotropical, 10(1), 133-139.

Weber, W., \& Rabinowitz, A. (1996). A global perspective on large carnivore conservation. Conservation Biology, 10, 1046-1054.

White, G. C., Anderson, D. R., Burnham, K. P., \& Otis, D. L. (1982). Capture-recapture and removal methods for sampling closed populations. Los Alamos, New Mexico, USA: Los Alamos National Laboratory.

Woodroffe, R., \& Ginsberg, J. R. (1998). Edge effects and the extinction of populations inside protected areas. Science, 280, 2126-2128.

Zamora, J. (2012). Manual Básico de Fototrampeo: Aplicaciones al estudio de los vertebrados terrestres. Valencia, España: Técnicas en Biología de la Conservación - $\mathrm{N}^{\circ} 4$. Tundra Ediciones.

Zeller, K. (2007). Jaguar in the New Millenium Data Set Update: The State of Jaguar in 2006. Wildlife Conservation Society's Jaguar Conservation Program.

Zimmermann, A., Walpole, M. J., \& Leaderwilliams, N. (2005). Cattle ranchers' attitudes to conflicts with jaguar (Panthera onca) in the Pantanal of Brazil. Fauna \& Flora International, Oryx, 39, 406-412. 